\title{
Creating Social-aware Evacuation Plans based on a GIS-enable Agent-based Simulation
}

\author{
Kasemsak Padungpien and Worawan Marurngsith \\ Department of Computer Science, Thammasat University, Thailand, wdc@cs.tu.ac.th
}

\begin{abstract}
In disaster preparedness, agent-based simulation (ABS) is an effective tool for aggregating information on evacuees affected by disasters. An agent usually imitates a household; and its actions are normally specified by decision models based on risk perception and social elements. Assigning socially connected households to the same shelters can better utilise resources. However, by pre-assigning specified regions to shelters, social connections are often omitted when developing evacuation plans at policy level. Thus, this paper presents a method to create social-aware evacuation plans. A GIS-enabled ABS is used to estimate evacuation demand and group evacuees according to their social connection data prior to assign them to the nearest shelters. The evacuation plans generated by the proposed method are compared against the travel-cost optimisation plans solved by using a linear model. The results obtained from a case study show that the social-aware evacuation plans offer a slightly better utilisation of shelter capacity; take similar time to evacuate households; yet could save nearly three hours to achieve complete evacuation. These results seem to confirm the competitiveness of social-aware evacuation plans as an option for evacuation planning at policy level.
\end{abstract}

Keywords: agent-based simulation, social connection, $K$-means clustering, linear programming, evacuation and shelter planning, GeoMASON

\section{Introduction}

Evacuation and shelter planning is essential to decrease the severity of loss and damage caused by either natural or man-made disasters. Evacuation studies may be classified into three groups: disaster models, traffic models, and traffic disaster evacuation models (Bae et al., 2014). Previous studies from 1985 - 2014 as reviewed in (Bae et al., 2014; NaBanerjee, 2015) confirmed that agent-based simulation (ABS) is the most popular tool used for gaining aggregated information of an evacuation. A finding has pinpointed that an evacuation decision is determined by a combination of household characteristics and by both capacity-related and hazard-related factors (Lim et al., 2016).

The first step in evacuation planning, is to forecast the number of people to be evacuated and the time to start evacuation, i.e. the demand estimation. ABS models were successfully used in (FlötterödLämmel, 2010; NaBanerjee, 2015; Yin et al., 2014) to gain insight into the evolution of evacuation demand when considering both risk factors and social elements. Proactive agents, i.e. goal-oriented agents searching for safe places or performing tasks to prepare for evacuation, were used to model households (NaBanerjee, 2015), individuals (FlötterödLämmel, 2010) and patients (Yin et al., 2014). The agents take actions based on the decision models integrated into a simulation (Gama et al., 2016; Lei et al., 2012; Lim et al., 2016; Yin et al., 2014). These models are mainly probabilistic decision models derived from human behaviour in response to hazards and evacuees' demographic characteristics (e.g. sex, age, education, social connection, and economical status) collected from historical records and interviews.

Evacuation plans obtained from aggregated results of ABS are not only determined by the shortest routes, but also by the behaviour of households based on their social connections (i.e., how friends and relatives might influence a household). Research efforts have successfully integrated some social connection factors into decision models as an attempt to better understand dynamic factors in an evacuation decision (Lim et al., 2016; Yin et al., 2014). Their findings confirmed the role of social connections among evacuees in effective emergency response, as described in (Hofinger et al., 2014; Wachtendorf et al., 2013). Aggregating agents' social interaction using ABS may not be applicable when creating plans based on regional segmentation. Besides, shelter assignment techniques based on regions are usually focussed on transportation optimisation rather than social connections. Consequently, social connections are often omitted when developing evacuation plans at policy level. 


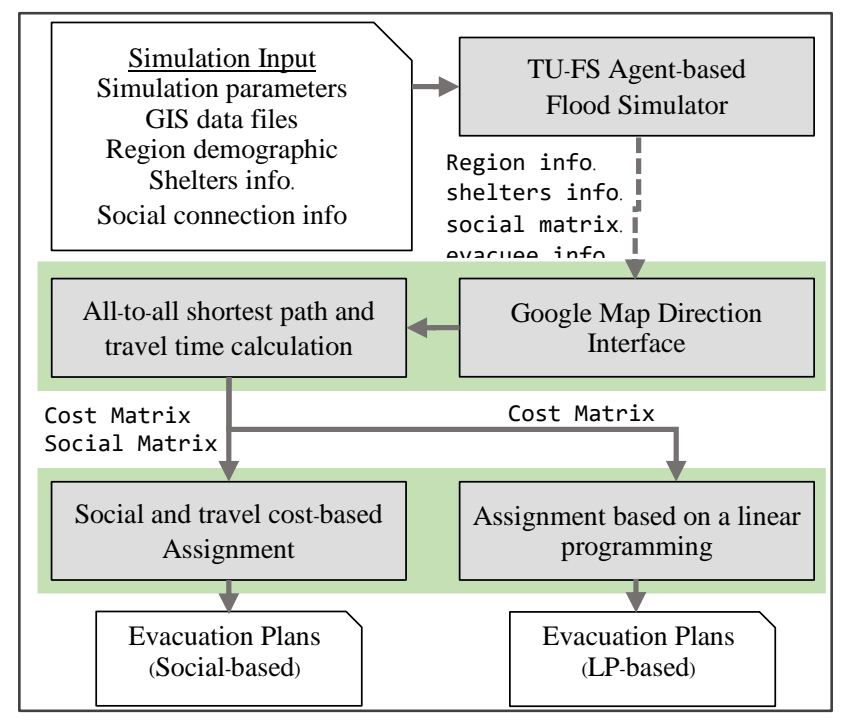

Figure 1. Overall process to generate the evacuation and shelter plans.

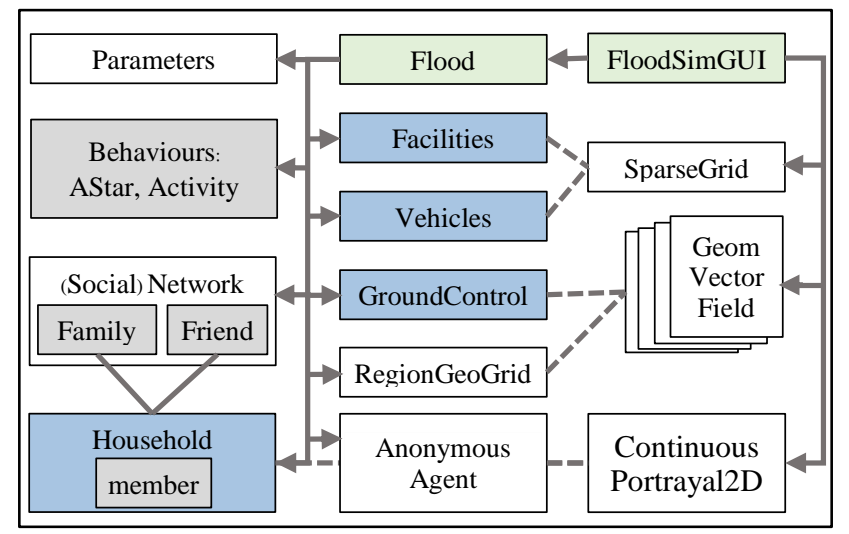

Figure 2. Classes and components in the TU-FS simulator.

\begin{tabular}{|c|c|c|c|c|c|c|c|c|c|c|}
\hline \multirow{2}{*}{$\begin{array}{c}\text { Input: } \\
\text { \#Population }\end{array}$} & \multirow[b]{2}{*}{ Region } & \multicolumn{4}{|c|}{ Family/Friend Counts } & \multicolumn{5}{|c|}{ Relationship Weight } \\
\hline & & \multicolumn{4}{|c|}{$\begin{array}{llll}\mathrm{T} 1 & \mathrm{~T} 2 & \mathrm{~T} 3 & \mathrm{~T} 4 \\
\end{array}$} & & $\mathrm{~T} 1$ & $\mathrm{~T} 2$ & \multirow{2}{*}{$\frac{\mathrm{T} 3}{0.20}$} & \multirow{2}{*}{\begin{tabular}{|l|}
\multicolumn{1}{|c}{ T4 } \\
0.05 \\
\end{tabular}} \\
\hline 100 & $\mathrm{~T} 1$ & 1 & 10 & 20 & 5 & $\mathrm{~T} 1$ & 1 & 0.10 & & \\
\hline 200 & $\mathrm{~T} 2$ & 80 & 1 & 25 & & & 0.40 & 1 & 0.13 & 0.00 \\
\hline 150 & $\mathrm{~T} 3$ & 30 & 20 & 1 & 5 & $\mathrm{~T} 3$ & 0.20 & 0.13 & 1 & 0.03 \\
\hline 200 & $\mathrm{~T} 4$ & 10 & 0 & 50 & 1 & $\mathrm{~T} 4$ & 0.05 & 0.00 & 0.25 & \\
\hline \multicolumn{11}{|c|}{-- After Simulation ------- } \\
\hline Output: & & \multicolumn{4}{|c|}{ Population relocation } & & \multicolumn{4}{|c|}{ Social Matrix } \\
\hline \#Population & Region & $\mathrm{T} 1 \mathrm{~T}$ & $\mathrm{~T} 2 \mathrm{~T}$ & $\mathrm{~T} 3 \mathrm{~T}$ & $\mathrm{~T} 4$ & & $\mathrm{~T} 1$ & $\mathrm{~T} 2$ & $\mathrm{~T} 3$ & $\mathrm{~T} 4$ \\
\hline 100 & $\mathrm{~T} 1$ & 80 & 5 & 10 & 5] & $\mathrm{T} 1$ & 1 & 0.25 & 0.5 & \begin{tabular}{|l|l|} 
\\
\end{tabular} \\
\hline 200 & $\mathrm{~T} 2$ & 40 & \begin{tabular}{|l|}
150 \\
\end{tabular} & 10 & 0 & & 0.8 & 1 & $\begin{array}{ll} \\
\end{array}$ & 0 \\
\hline 150 & $\mathrm{~T} 3$ & \begin{tabular}{l|l|}
30 \\
\end{tabular} & \begin{tabular}{|l|}
0 \\
\end{tabular} & 80 & 20 & $\mathrm{~T} 3$ & \begin{tabular}{|l|}
0.43 \\
\end{tabular} & 0 & 1 & 0.29 \\
\hline 200 & $\mathrm{~T} 4$ & 10 & 0 & 0 & 180 & $\mathrm{~T} 4$ & 0.5 & 0 & 0 & 1 \\
\hline
\end{tabular}

Figure 3. Example of the social connection and the social matrix.

This paper presents a method to create a social-aware evacuation plan. A GIS-enabled ABS called the TU-FS simulator (Vijitpornkul and Marurngsith, 2015), implemented on the GeoMASON framework (George Mason University, 2013), is used to estimate evacuation demand and obtain social connection data. These data are used to classify evacuees into groups and assign them to the nearest shelters. This paper makes three contributions.

(1) An estimation of evacuation demands and households' social connections using a GIS-enabled agent-based flood simulator is demonstrated.

(2) A shelter assignment method to generate socialaware evacuation plans by using the K-means clustering and realistic traffic information obtained via the Google Map Direction API (Google Developer, 2016) is proposed.

(3) A linear optimisation model for solving the capacity-aware shelter assignment problem based on minimising travel-cost is presented. Evacuation plans generated by this model were compared against the social-aware plans and this confirmed the effectiveness of the proposed social-aware method.

A case study, covering the central part of Lop Buri province in Thailand, was conducted to demonstrate the applicability of the proposed method. The results confirm the competitiveness of social-aware evacuation plans as an appropriate alternative to current policies for evacuation.

The rest of this paper is organised as follows. The next section introduces an agent-based flood simulation used in this study. Section 3 presents the proposed evacuation and shelter planning method. Section 4 analyses the efficiency gained from allocating ten shelters with the proposed method in comparison to the linear optimisation model. Finally, Section 5 brings this paper to a conclusion.

\section{GIS-Enabled Agent-Based Flood Simulation}

The overall process to generate evacuation and shelter plans is shown in Figure 1. Firstly, users must setup the flood scenarios by providing six inputs i.e., (1) the simulation parameters, (2) the GIS data files, (3) information about the affected regions, (4) information about shelters and other facilities, (5) social connection information and (6) the household demographics. All these inputs are then passed to the TU-FS simulator to run a simulation. The TU-FS simulator gathers the amount of population in the affected regions and processes it into four output files that represent the evacuation demand. These four files contain information about the affected regions, shelters, households (evacuees), and a social matrix, respectively. Data contained in those four files is processed using the Google Map Direction interface followed by an all-to-all shortest path and travel time calculation. The process creates two matrices, namely: a cost matrix, and a social matrix. In our approach, we use both matrices to assign shelters and generate evacuation plans. In contrast, the linear model only uses the cost matrix to deal with shelter-assignment and to produce evacuation plans. 
We have modified the original TU-FS simulator to be able to simulate pre-evacuation activity in different scenarios. The core simulation model and the graphical user interface of the model were implemented in two Java classes: Flood and FloodSimGUI (Figure 2). The Flood class contains objects from four steppable classes (Facility, Vehicles, GroundControl, and Household), four custom classes (AStar, Activity, Family, Friend) and other objects inherited from the MASON and GeoMASON libraries. The steppable classes implement objects whose behaviour is updated at every time step during a simulation. Custom classes are used to implement specific behaviours of the objects. For example, the AStar class implements an algorithm to assign a household or a vehicle to a certain travel path. Four objects extended from the Mason library are used to enable customised parameters. These are the ContinuousPortrayal2D, SparseGrid, Network, and Param. These objects are also used for creating both the social network relationship among households and the layered environments in the model. GIS data files are added into the model using two classes of the GeoMASON library, i.e., the GeomVectorfield and RegionGeoGrid objects. Details of the MASON simulation engine can be found in (Luke, 2015).

The GroundControl class manages the GIS data layers in the model. To do this, three parameters are required, namely: a list of GIS ASCII format files, the affected region and shelter location. The GIS layers of an area under study are read from the list of GIS ASCII files and placed on top of the 2D continuous space containing the households' agents. These layers represent the political boundary of a region, the ground water, the transportation (road network), and the flood map. The flood map layer consists of a set of files that are named in sequence. That map is updated at every specified simulation time step (e.g. every simulated hour). The TU-FS simulator uses the WGS84 (EPSG4326) coordinate reference system. The list of regions and shelter locations is stored in a CSV and fed into the simulation by the GroundControl class. The GPS locations are also sent to the Facilities class to create agents for shelters and sub-districts.

Parameters related to household demographics and social connections are also specified in the CSV-format files. The household demographics file records the number of affected regions or sub-districts under study. That information is kept in rows, where every row specifies information about the households in a subdistrict as the percentage of the population having certain attributes. For example, these attributes could be personal characteristics (sex, height, average age), health status (healthy, infected, sick, dead), number of families, mobility state. The parameters used to derive social connections are in the FriendInfo and FamilyInfo files. These files contain 2D matrices that represent a Count and a Size part. Each part of the file has a $2 \mathrm{D}$ matrix of $\mathrm{R} \times \mathrm{R}$ size, where $\mathrm{R}$ is the number of the affected regions. Figure 3. (top left) depicts the structure of the Count part of the file. The Size part is kept in a matrix where each cell (in row $i$ column $j$ ) represents the average of friend/family members that live in sub-district $\mathrm{j}$ of a person that lives in sub-district i. The FriendInfo and FamilyInfo files are used to create Household, Family, Friend and Vehicle objects to meet the specified parameters. Social network relationships are set using the MASON's social network facility.

Once all objects have been successfully initialised, the TU-FS simulator runs a simulation until either a defined step or a trigger marked in the flood map is reached. At every step in the simulation, household agents may move to join with friends or family. The simulation summarises locations of agents and other detailed information into four text files (Figure 1). At this stage, the number of households who live in subdistrict $i$ but have moved and stay in sub-district $j$ is recorded in cell $i, j$ of the population relocation matrix (see Figure 3 lower left). Data in this relocation matrix is calculated as a percentage and written into a SocialMatrix file (Figure 3 lower right). Finally, all four output files are used by the shelter allocation module to produce an evacuation plan.

\section{Evacuation and Shelter Planning Method}

\subsection{Shelter Allocation Methodology}

As depicted in Figure 1, the shelter allocation module takes the output of the ABS module and performs four key steps. The detail algorithm is presented in Table 1. Firstly, we use a web service from the Google Direction API to obtain three driving routes with their directions as a JASON object. The routes are extracted from the JASON object and stored in a origin/destination matrix, the OD matrix. Any unsafe roads obtained from the simulation are rejected as feasible routes.

Secondly, the travel time from all sub-districts to every shelter is calculated prior to applying the FloydWarshall algorithm to find the all-to-all shortest path. The calculation of travel time is based on the simple carfollowing evacuation model, but including the travel speed obtained from the Google Map Direction API. The RegionInfo file contains a list of vehicles that are used to calculate the length of vehicle processions for each affected region. After that, speed and distance in the OD matrix are used to calculate the overall evacuation time of all vehicle processions from each affected region to shelters. The results containing the evacuation times are stored in a new matrix called the cost matrix (C). 
Table 1. Algorithm used in The Shelter Allocation Module.

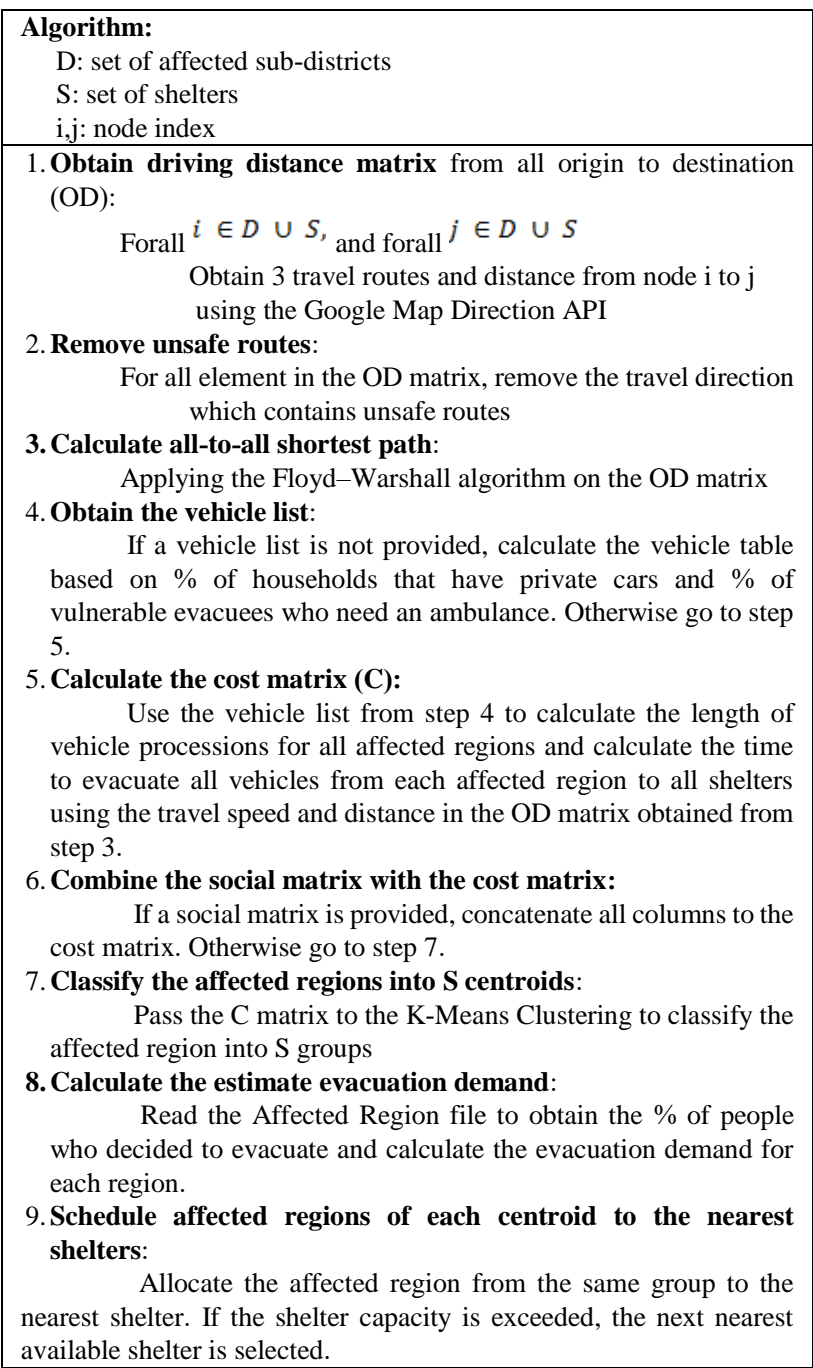

\subsection{Shelter Assignment based on K-Means Clustering}

The third key step performs the travel-cost and socialbased shelter assignment. In this step, affected regions are classified into $\mathrm{S}$ groups based on travel time and social connections, where $\mathrm{S}$ is the number of shelters. The combination of the Social matrix and the $\mathrm{C}$ matrix is used as the input features for the K-Means Clustering module. Ideally, all groups of an affected region will be assigned to common shelters.

To assign shelters to affected regions the number of evacuees is first estimated by applying the percentage of people that decided to go to shelters, relative to the initial population. To proceed with allocation, the system will try to assign an affected region from a group to the nearest shelter allocated to that group, i.e. the Our Shelter. If the Our Shelter cannot accommodate any more evacuees, the next nearest available shelter with the closest social connection will be selected, i.e. the Our Friend Shelter. The latter selection process is iteratively applied until an available shelter is found.

\subsection{Linear Optimisation Model}

The last key step in the shelter allocation is to generated alternative evacuation plans by using a linear optimisation model. To solve shelter assignment, we have implemented a linear optimisation model based on minimising travel time using the Simplex method. Parameters and decision variables in our model, are defined as follows.

$\mathrm{S} \quad$ set of shelters

D set of sub-districts to be evacuated

I index of sub-district

$\mathrm{J}$ index of shelter

$\mathrm{Ei}$ the number of evacuees in sub-district $\mathrm{i}$

$\mathrm{Lj}$ the capacity limit of shelter $\mathrm{j}$

$\mathrm{Cij}$ the evacuation time

Decision variable:

$x_{i j}$ is $\left\{\begin{array}{l}x_{i j} \geq 1 \text { and } x_{i j} \leq \mathrm{E}_{\mathrm{i}} \text { if subdistrict } \mathrm{i} \text { is assigned to shelter } \mathrm{j} \\ 0 \text { otherwise }\end{array}\right.$

Objective function:

$$
\min \sum_{i \in D} \sum_{j \in S} x_{i j} C_{i j}
$$

Subject to:

$$
\begin{aligned}
& \sum_{j \in S} x_{i j}=E_{i} \\
& \sum_{i \in D} x_{i j} \leq L_{j}
\end{aligned}
$$

In our model, the number of evacuees of sub-district $i$ assigned to shelter $\mathrm{j}$ is used as a decision variable. The objective function, Equation (1), minimises total evacuation time based on travel cost to evacuate all evacuees from all sub-districts to their assigned shelters. The constraint in Equation (2) guarantees that the number of evacuees of a sub-district assigned to different shelters is equal to the total number of evacuees in that sub-district. The constrain in Equation (3), ensures that the number of evacuees from subdistricts assigned to a shelter does not exceed that shelter's capacity.

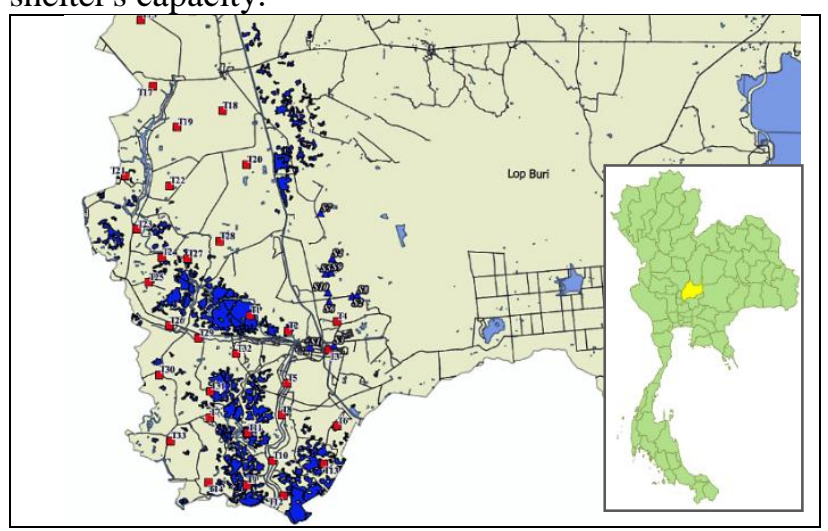

Figure 4. Screenshot showing the studied area in Lop Buri, Thailand 


\section{Case Study}

A case study was carried out to demonstrate the applicability of the proposed method. The case study is located in the centre of Lop Buri province, Thailand, covering $6,200 \mathrm{~km}^{2}$ area. The information used in the case study is based on the Lop Buri's official evacuation and shelter plan of the year 2011. The official plan specified 33 sub-districts or Tambon (T1 - T33 shown in Table 2) with a potential flood-affected population of 149,468 people living in 8,300 households. Furthermore, there were 1,939 vulnerable people among the affected population. Ten potential public shelters were pre-defined with capacities of $500-3,150$ people (see S1 - S10 in Table 3). Those shelters were existing public schools, hospitals, and military facilities located on high ground. Flood maps and geographical information from the Thailand Flood Monitory System provided by the Geo-Informatics and Space Technology Development Agency (GISTDA, 2016) were used to locate shelters and the affected regions (Figure 4).

The progression of the flood was simulated based on the flood map of the year 2012. To investigate advantages and drawbacks of our approach we compare the generated social-aware evacuation plans (S-plans) against their counterparts generated from the linear programming optimisation (LP-plans). We considered four scenarios (see below) that produced eight different plans: (S-A1, -A2, -B1, -B2 and LP-A1, -A2, -B1, -B2).

A1: The evacuation is performed before the inundation has progressed to the potential affected regions. Healthy evacuees use private vehicles to travel to the assigned shelters.

A2: As for A1, except that public buses are used to transport all healthy evacuees.

B1: The evacuation is started when some roads are already inundated and unsafe to use. Healthy evacuees use private vehicles to travel to the assigned shelters.

B2: As for B1, except that public buses are used to transport all healthy evacuees.

Note that ambulances were used to evacuate vulnerable evacuees in all experimental settings. The result from the ABS shows that 10 percent of the population will be evacuated. This conform with the findings in (Lei et al., 2012; Yin et al., 2014).

\subsection{Shelter Utilisation}

From the perspective of capacity utilisation, both the social-aware and LP-plans achieved very similar results (Figure 5) ranging from $78-81.5$ percent. In the LP plans, we observed that some shelters were packed up to $100 \%$ to minimise the evacuation time. However, other shelters were not used at all. Thus, the social-aware plans show a slightly better (by $1 \%-2 \%$ ) shelter utilisation.

\subsection{Time to Evacuate One Household}

In the generated plan, the time to evacuate one household is reported (an example of the generated plan is shown in Table 4). In all studied scenarios a household needs on average about an hour ( -6 to +20 min.) to arrive at its assigned shelter (Figure 6). Although the linear optimised plans show less evacuation time per household, they have a wider range of min-max time. The most effective evacuation is observed in the S-A1 plan in which all evacuees could arrive at their assigned shelters in one hour and twentysix minutes. The worst time is shown by the S-B1 plan as road cuts make a trip time of three hours and twentyone minutes.

Table 2. The Demographic of Flood-Affected Regions.

\begin{tabular}{|l|l|l|l|l|}
\hline ID & Sub-district & Household & Population & Vulnerable \\
\hline T1 & Bang Khan Mak & 2,462 & 9,811 & 131 \\
\hline T2 & Phrommat & 1,275 & 4,563 & 45 \\
\hline T3 & Tha Hin & 1,655 & 4,629 & 0 \\
\hline T4 & Tha Le Chup & 2,230 & 9,091 & 116 \\
& Sorn & & & \\
\hline T5 & Pho Kao Ton & 4,345 & 14,834 & 95 \\
\hline T6 & Khok Lamphan & 844 & 3,596 & 62 \\
\hline T7 & Pho Tru & 661 & 2,395 & 33 \\
\hline T8 & Talung & 1,051 & 4,234 & 50 \\
\hline T9 & Si Khlong & 258 & 993 & 25 \\
\hline T10 & Ngiu Rai & 1,337 & 5,546 & 103 \\
\hline T11 & Thai Talat & 880 & 3,477 & 45 \\
\hline T12 & Kong Thanu & 1,276 & 4,301 & 77 \\
\hline T13 & Don Pho & 876 & 4,012 & 23 \\
\hline T14 & Ban Khoi & 741 & 2,912 & 68 \\
\hline T15 & Phai Yai & 1,016 & 4,174 & 60 \\
\hline T16 & Sai Huai Kaeo & 530 & 2,287 & 47 \\
\hline T17 & Mahason & 744 & 3,069 & 48 \\
\hline T18 & Sanam Chaeng & 1,222 & 6,070 & 0 \\
\hline T19 & Bang Phung & 1,280 & 4,555 & 27 \\
\hline T20 & Nong Tao & 928 & 4,087 & 76 \\
\hline T21 & Bang Kham & 772 & 3,379 & 61 \\
\hline T22 & Ban Chi & 1,232 & 5,486 & 74 \\
\hline T23 & Khao Samo Khon & 1,149 & 4,678 & 110 \\
\hline T24 & Khok Salut & 500 & 1,912 & 37 \\
\hline T25 & Bang Nga & 769 & 3,073 & 31 \\
\hline T26 & Tha Wung & 594 & 2,075 & 70 \\
\hline T27 & Muchalin & 639 & 2,508 & 54 \\
\hline T28 & Bang Li & 1,082 & 4,519 & 96 \\
\hline T29 & Bang Khu & 407 & 1,438 & 24 \\
\hline T30 & Hua Samrong & 1,693 & 6,467 & 110 \\
\hline T31 & Lat Sali & 845 & 3,555 & 70 \\
\hline T32 & Pho Talat Kaeo & 1,243 & 4,507 & 71 \\
\hline T33 & Ban Boek & 1,764 & 5,296 & 0 \\
\hline & & & \\
\hline
\end{tabular}

Table 3. Shelters Information.

\begin{tabular}{|l|l|l|}
\hline ID & Shelters & Capacity \\
\hline S1 & Khai Narai Suksa Royal Thai Army School & 3,150 \\
\hline S2 & Jirawichit Songkhram Camp & 3,150 \\
\hline S3 & The 13th Military Circle & 1,050 \\
\hline S4 & Artillery Center & 2,100 \\
\hline S5 & Artillery Division & 2,100 \\
\hline S6 & The 31st Infantry Regiment King's Guard & 1,575 \\
\hline S7 & Army Aviation & 2,100 \\
\hline S8 & Ananda Mahidol Hospital & 1,050 \\
\hline S9 & Wing 2 & 1,575 \\
\hline S10 & The 11th Artillery Battalion King's Guard & 525 \\
\hline
\end{tabular}


Table 4. Example of The Generated Plan (S-A1).

\begin{tabular}{|c|c|c|c|c|l|l|}
\hline From & To & $\begin{array}{l}\text { Time } \\
\text { (h:m:s) }\end{array}$ & $\begin{array}{c}\text { Distanc } \\
\mathbf{e} \\
\text { (km.) }\end{array}$ & Evacuees & \multicolumn{2}{|l|}{ Route } \\
\hline T6 & S1 & $0: 21: 49$ & 11.5 & 361 & R.3016 & \\
\hline T8 & S1 & $0: 18: 42$ & 9.4 & 425 & R.3196 & \\
\hline T10 & S1 & $0: 23: 29$ & 15.7 & 557 & R.3196 & \\
\hline T11 & S1 & $0: 24: 21$ & 14.9 & 349 & R.5071 & \\
\hline T13 & S1 & $0: 18: 39$ & 17.9 & 402 & R.3196 & \\
\hline T14 & S1 & $0: 30: 13$ & 22.1 & 291 & R.311 & \\
\hline$:$ & $:$ & $:$ & $:$ & $:$ & $:$ & $:$ \\
$:$ & $:$ & $:$ & $:$ & $:$ & $:$ & $:$ \\
\hline T16 & S4 & $0: 27: 40$ & 34.5 & 229 & R.3196 & \\
\hline T17 & S4 & $0: 29: 06$ & 31.5 & 308 & R.3196 & \\
\hline T18 & S4 & $0: 26: 21$ & 23 & 607 & R.3196 & \\
\hline T31 & S4 & $0: 36: 54$ & 26.5 & 358 & R.1 & \\
\hline T5 & S5 & $0: 36: 30$ & 16.3 & 1488 & R.3196 & R.1 \\
\hline T12 & S6 & $0: 33: 21$ & 28.9 & 431 & R.3196 & \\
\hline T3 & S7 & $0: 32: 35$ & 17.4 & 463 & R.1 & \\
\hline T4 & S7 & $0: 34: 28$ & 13.4 & 912 & R.1 & \\
\hline T27 & S7 & $0: 26: 51$ & 22.1 & 253 & R.3019 & \\
\hline T28 & S7 & $0: 27: 44$ & 14.6 & 453 & R.3019 & \\
\hline T33 & S7 & $0: 44: 07$ & 36.3 & 530 & R.3027 & \\
\hline T30 & S8 & $0: 35: 25$ & 28 & 651 & R.3027 & \\
\hline T20 & S9 & $0: 33: 18$ & 16.7 & 411 & R.3196 & R.1 \\
\hline T23 & S9 & $0: 20: 32$ & 29 & 469 & R.1 & \\
\hline T32 & S9 & $0: 21: 22$ & 20.8 & 453 & R.311 & R.1 \\
\hline T19 & S10 & $0: 18: 55$ & 30.3 & 458 & R.3196 & \\
\hline T1 & S10 & $0: 35: 12$ & 19.1 & 985 & R.3196 & \\
\hline & & & & & & \\
\hline
\end{tabular}

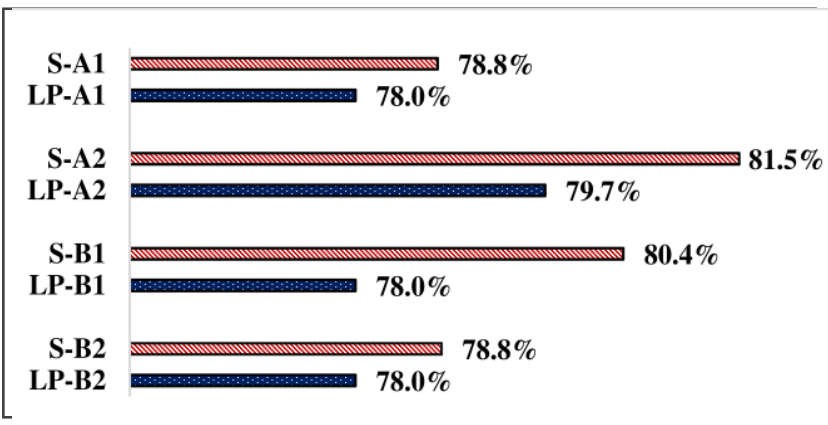

Figure 5. Average shelter utilisation of social-vs. LP-plans.
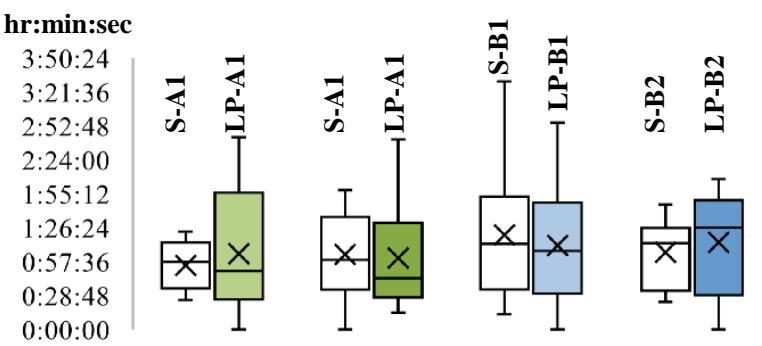

Figure 6. Time to evacuate a household to the assigned shelter.

\subsection{Total Evacuation Time}

The overall evacuation time is analysed from both a best case and a worst-case perspective. In the former case, termed the minimum travel time, traffic congestion was taking into account and processions of evacuees' vehicles, going to different shelters and following different routes, were scheduled in parallel. In the latter case, termed the maximum travel time, processions of evacuees' vehicles were moved in sequence to mimic heavy traffic congestion.

Travel time differences between social-aware and LPplans are shown in Figure 7 and Figure 8. Note that positive values indicate that a social-aware plan is faster than its LP plan counterpart. Our results show that in comparison to the LP plans, all S-plans (with the exception of the minimum time of S-A1) save an average of between half an hour and up to three hours of travel time. Obviously, the travel time required for evacuation depends on the conditions of the route selected, which takes its toll in the slower minimum travel time of the S-A1 plan.

\subsection{Discussion}

Our approach capitalises on the detailed information on distances and available routes to produce social aware evacuation plans that seem to be more capable than simple LP plans. Note that our methodology manages to make evacuation plans even in the event of road cuts, providing that those road cuts do not cause isolation areas.

\section{Conclusions and Future Work}

In this paper, a GIS-enabled agent based simulation to create social-aware evacuation plans, based on estimation of household movement during flood, has been presented. The implementation of an updated version of the TU-FS simulator and the shelter assignment method have been described. A case study has been conducted to demonstrate the applicability of the proposed method. Two evacuation scenarios were considered: evacuation prior to the arrival of a flood, and evacuation during a low-level flood. The evacuation plans generated by the proposed method were compared against their linear model travel-cost optimisation counterparts. The results show that plans generated with our methodology offer a 1-2\% better use of shelter capacity. The average evacuation times per household are similar to those of optimised plans with a difference between $-12 \%$ to $+15 \%$. However, our evacuation plans could save nearly three hours to evacuate all regions.

Thus, the methodology presented in this paper could be an appropriate alternative to develop evacuation plans at policy level. Future work will focus on improving the social matrix model and model verification.

\section{Acknowledgements}

We thank GISTDA for the GIS data files used in this project. We thank the reviewers for their valuable comments. We thank contributors to the MASON and 


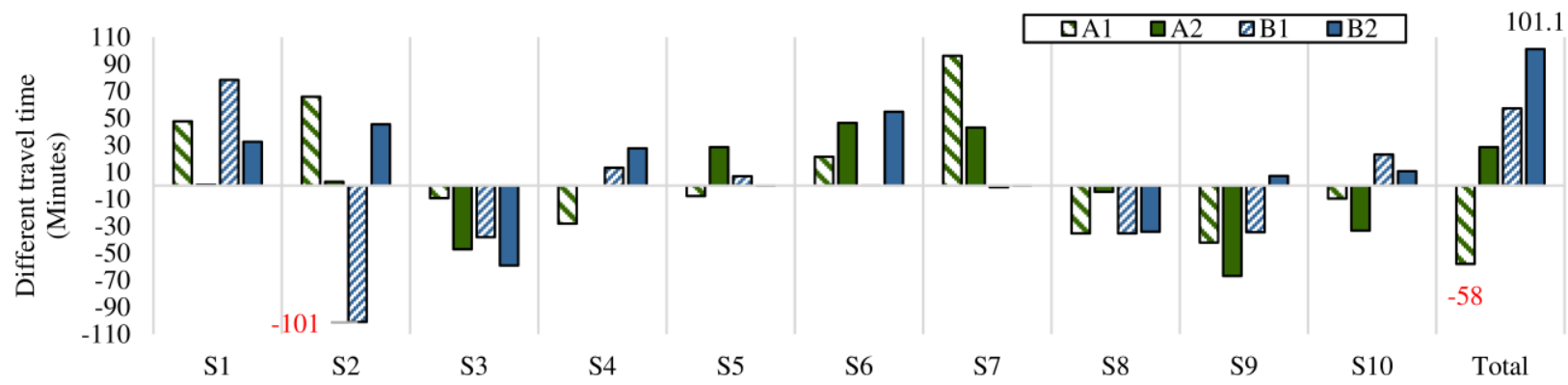

Figure 7. Difference of minimum travel time between the Social- and the LP- plans (positive value = the Social Plan is faster for $\mathrm{x} \min )$.

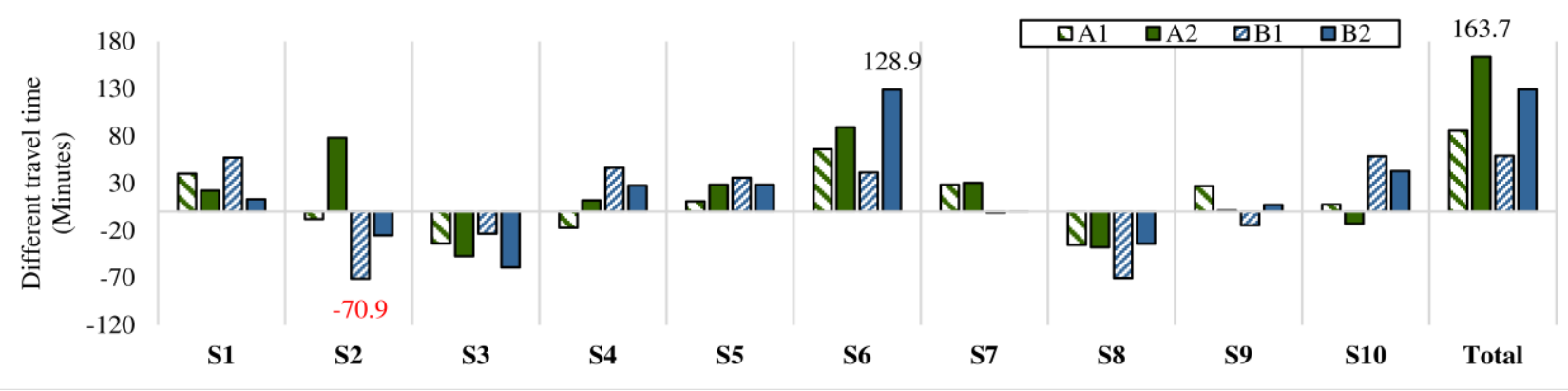

Figure 8. Difference of maximum travel time between Social- and the LP- plans (positive value = the Social Plan is faster for $\mathrm{x}$ minutes).

GeoMASON community website for discussion and lesson learned. We thank Professor Roland Ibbett and JC Diaz Carballo for improving the readability of this paper.

\section{References}

J. W. Bae, S. Lee, J. H. Hong, and I. C. Moon. Simulationbased analyses of an evacuation from a metropolis during a bombardment. Simulation, 90(11): 1244-1267, 2014.

G. Flötteröd and G. Lämmel. Evacuation simulation with limited capacity sinks: An evolutionary approach to solve the shelter allocation and capacity assignment problem in a multi-agent evacuation simulation. In ICEC 2010 Proceedings of the International Conference on Evolutionary Computation, 2010.

M. Gama, B. Santos, and M. Scaparra. A multi-period shelter location-allocation model with evacuation orders for flood disasters. EURO Journal on Computational Optimization, 4(3-4): 299-323, 2016.

George Mason University. GeoMason: GeoSpatial Support for MASON. [accessed 2013].

GISTDA. Thailand Flood Monitoring System. Available via http://flood.gistda.or.th/ [accessed 2016].

Google Developer. Google Map Direction API. Available via https://developers.google.com/maps/documentation/directions/ [accessed 2016].
G. Hofinger, R. Zinke, and L. Künzer. Human Factors in Evacuation Simulation, Planning, and Guidance. Transportation Research Procedia, 2: 603-611, 2014.

D. Lei, W. Wenjun, and Z. Xiankun. An agent-based decisionmaking model in emergency evacuation management. Journal of Convergence Information Technology, 7(10): 197-205, 2012.

M. B. B. Lim, H. R. Lim, M. Piantanakulchai, and F. A. Uy. A household-level flood evacuation decision model in Quezon City, Philippines. Natural Hazards, 80(3): 15391561, 2016.

S. Luke. Multiagent Simulation and the MASON Library. Available via https://cs.gmu.edu/ eclab/projects/mason/.

H. S. Na and A. Banerjee. An agent-based discrete event simulation approach for modeling large-scale disaster evacuation network.In Proceedings - Winter Simulation Conference, 2015.

S. Vijitpornkul and W. Marurngsith. Simulating crowd movement in agent-based model of large-scale flood.In Advanced Informatics: Concepts, Theory and Applications (ICAICTA), 2015 2nd International Conference on, 2015.

T. Wachtendorf, M. M. Nelan, and L. Blinn-Pike. Households and Families. In Social Vulnerability to Disasters, Second Edition: Taylor \& Francis, 2013.

W. Yin, P. Murray-Tuite, S. V. Ukkusuri, and H. Gladwin. An agent-based modeling system for travel demand simulation for hurricane evacuation. Transportation Research Part C: Emerging Technologies, 42: 44-59, 2014. 